\title{
Severity-associated markers and assessment model for predicting the severity of COVID-19: a retrospective study in Hangzhou, China
}

Jianjiang $\mathrm{Qi}^{1 \dagger}$, Di He ${ }^{2 \dagger}$, Dagan Yang ${ }^{3 \dagger}$, Mengyan Wang ${ }^{1}$, Wenjun Ma ${ }^{1}$, Huaizhong Cui ${ }^{1}$, Fei Ye ${ }^{1}$, Fei Wang ${ }^{1}$,

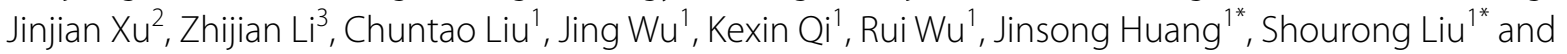
Yimin $Z_{h}{ }^{2,4^{*}}$

\begin{abstract}
Background: The severity of COVID-19 associates with the clinical decision making and the prognosis of COVID-19 patients, therefore, early identification of patients who are likely to develop severe or critical COVID-19 is critical in clinical practice. The aim of this study was to screen severity-associated markers and construct an assessment model for predicting the severity of COVID-19.

Methods: 172 confirmed COVID-19 patients were enrolled from two designated hospitals in Hangzhou, China. Ordinal logistic regression was used to screen severity-associated markers. Least Absolute Shrinkage and Selection Operator (LASSO) regression was performed for further feature selection. Assessment models were constructed using logistic regression, ridge regression, support vector machine and random forest. The area under the receiver operator characteristic curve (AUROC) was used to evaluate the performance of different models. Internal validation was performed by using bootstrap with 500 re-sampling in the training set, and external validation was performed in the validation set for the four models, respectively.
\end{abstract}

Results: Age, comorbidity, fever, and 18 laboratory markers were associated with the severity of COVID-19 (all $P$ values $<0.05$ ). By LASSO regression, eight markers were included for the assessment model construction. The ridge regression model had the best performance with AUROCs of $0.930(95 \% \mathrm{Cl}, 0.914-0.943)$ and $0.827(95 \% \mathrm{Cl}, 0.716$ 0.921 ) in the internal and external validations, respectively. A risk score, established based on the ridge regression model, had good discrimination in all patients with an AUROC of 0.897 ( $95 \% \mathrm{Cl} 0.845-0.940$ ), and a well-fitted calibration curve. Using the optimal cutoff value of 71 , the sensitivity and specificity were $87.1 \%$ and $78.1 \%$, respectively. A web-based assessment system was developed based on the risk score.

Conclusions: Eight clinical markers of lactate dehydrogenase, C-reactive protein, albumin, comorbidity, electrolyte disturbance, coagulation function, eosinophil and lymphocyte counts were associated with the severity of COVID-19.

*Correspondence: 13306817568@163.com; Isr85463990@126.com;

zhuym@zju.edu.cn

${ }^{\dagger}$ Jianjiang Qi, Di He and Dagan Yang authors contributed equally to this

work

${ }^{1}$ Hangzhou Xixi Hospital, Hangzhou 310023, Zhejiang, China

2 Department of Epidemiology \& Biostatistics, School of Public Health,

Zhejiang University, Hangzhou 310058, Zhejiang, China

Full list of author information is available at the end of the article

(c) The Author(s) 2021. Open Access This article is licensed under a Creative Commons Attribution 4.0 International License, which permits use, sharing, adaptation, distribution and reproduction in any medium or format, as long as you give appropriate credit to the original author(s) and the source, provide a link to the Creative Commons licence, and indicate if changes were made. The images or other third party material in this article are included in the article's Creative Commons licence, unless indicated otherwise in a credit line to the material. If material is not included in the article's Creative Commons licence and your intended use is not permitted by statutory regulation or exceeds the permitted use, you will need to obtain permission directly from the copyright holder. To view a copy of this licence, visit http://creativecommons.org/licenses/by/4.0/. The Creative Commons Public Domain Dedication waiver (http://creativeco mmons.org/publicdomain/zero/1.0/) applies to the data made available in this article, unless otherwise stated in a credit line to the data. 
An assessment model constructed with these eight markers would help the clinician to evaluate the likelihood of developing severity of COVID-19 at admission and early take measures on clinical treatment.

Keywords: COVID-19, Severity, Assessment model, Prediction, Web-based assessment system

\section{Background}

Coronavirus disease 2019 (COVID-19) is caused by severe acute respiratory syndrome coronavirus 2 (SARS-CoV-2) and has spread worldwide [1]. On March 12, 2020, the World Health Organization (WHO) announced the disease to be pandemic. It has affected more than 200 countries with about 10,000,000 confirmed cases as of July 01, 2020 [2]. Therefore, the epidemic of COVID-19 has become a global public health crisis.

Different clinical patterns, such as mild, moderate, and severe to critical types, were observed in patients with COVID-19. Although most COVID-19 patients have mild or moderate symptoms and signs, the finding from China indicated that about $14 \%$ of patients were of the severe type and 5\% were of the critical type [3]. Previous studies and clinical practice showed that the degree of severity was associated with the clinical treatment and prognosis of the disease [3-6]. The average overall case-fatality rate of confirmed COVID-19 patients was $2.3 \%$, but that was up to $49.0 \%$ in critical patients [3]. Missed diagnoses will delay the appropriate clinical treatment and increase the possibility of poor prognosis. On the other hand, treatment for a severe or critical COVID-19 patient requires vast medical resources, and over misdiagnoses will overuse the medical resources and increase the medical burden. Therefore, early identification of patients who are likely to develop severe or critical COVID-19 is especially important for clinical practice and epidemic control. In clinical practice, the severity of COVID-19 is categorised into four levels as mild, moderate, severe, and critical types according to the Seventh Edition of the Guide to Diagnosis and Treatment of New Coronary Pneumonia [7]. This classification is preformed mainly based on the clinical symptoms, oxygen saturation $(\mathrm{SaO} 2)$, and imaging evidence from computed tomography (CT). However, no evidence from laboratory markers has been included. Previous studies have found that lymphopenia, organ dysfunction, coagulopathy, and elevated $\mathrm{D}$-dimer levels were associated with the severity [3-6, 8].

In this study, we aimed to screen severity-associated markers and construct an assessment model for predicting the severity of patients with COVID-19 based on the data from two hospitals in Hangzhou, Zhejiang province, China.

\section{Methods}

\section{Study population}

This study enrolled 172 confirmed COVID-19 patients from January 20, 2020 to April 1, 2020 in Hangzhou, Zhejiang Province, China. Among these patients, 104 from Hangzhou Xixi Hospital were used for screening the severity-associated markers and constructing the assessment model as a training set. Part of the 104 patients had been used in the previously published studies [9, 10]. On the other hand, 68 patients from the First Affiliated Hospital, School of Medicine, Zhejiang University (FAHZJU) were used to validate the model as a validation set. These patients were part of the sample which had been published previously [10]. COVID-19 was diagnosed according to the interim guidance from the WHO [11]. The severity of COVID-19 was categorised into four levels according to the Seventh Edition of the Guide to Diagnosis and Treatment of New Coronary Pneumonia [7]. The mild type was defined as patients with mild clinical symptoms and normal imaging on CT. The moderate type was defined as patients with fever, respiratory symptoms, or other symptoms, and altered imaging evidence with pneumonia. The severe type was defined as patients with at least one of the following symptoms: shortness of breath (breathing rate $\geq 30 / \mathrm{min}$ ), $\mathrm{SaO}_{2}$ at rest $\leq 93 \%$, partial pressure of oxygen in arterial blood $\left(\mathrm{PaO}_{2}\right) /$ inspired oxygen fraction $\left(\mathrm{FiO}_{2}\right) \leq 300 \mathrm{mmHg}$, or lung infiltrates $>50 \%$ within 24 to $48 \mathrm{~h}$. The critical type was defined as patients with any of the following symptoms: respiratory failure requiring mechanical ventilation, shock, or a combination of other organ failures requiring ICU monitoring treatment.

This was a retrospective study and the protocol was approved by the Ethics Committee of Xixi Hospital and FAHZJU.

\section{Data collection}

Data at admission, including demographic information, comorbidities, clinical symptoms and laboratory tests, were extracted from electronic medical records. Collected data were reviewed by a trained team of clinical physicians. Demographic information included age, sex and body mass index (BMI). Comorbidity was defined as having at least one of the following diseases: diabetes, hypertension, cardiovascular disease, severe congenital disease, cancer, and chronic diseases of the liver, kidney, or respiratory system. Clinical symptoms included fever, fatigue, cough, expectoration, 


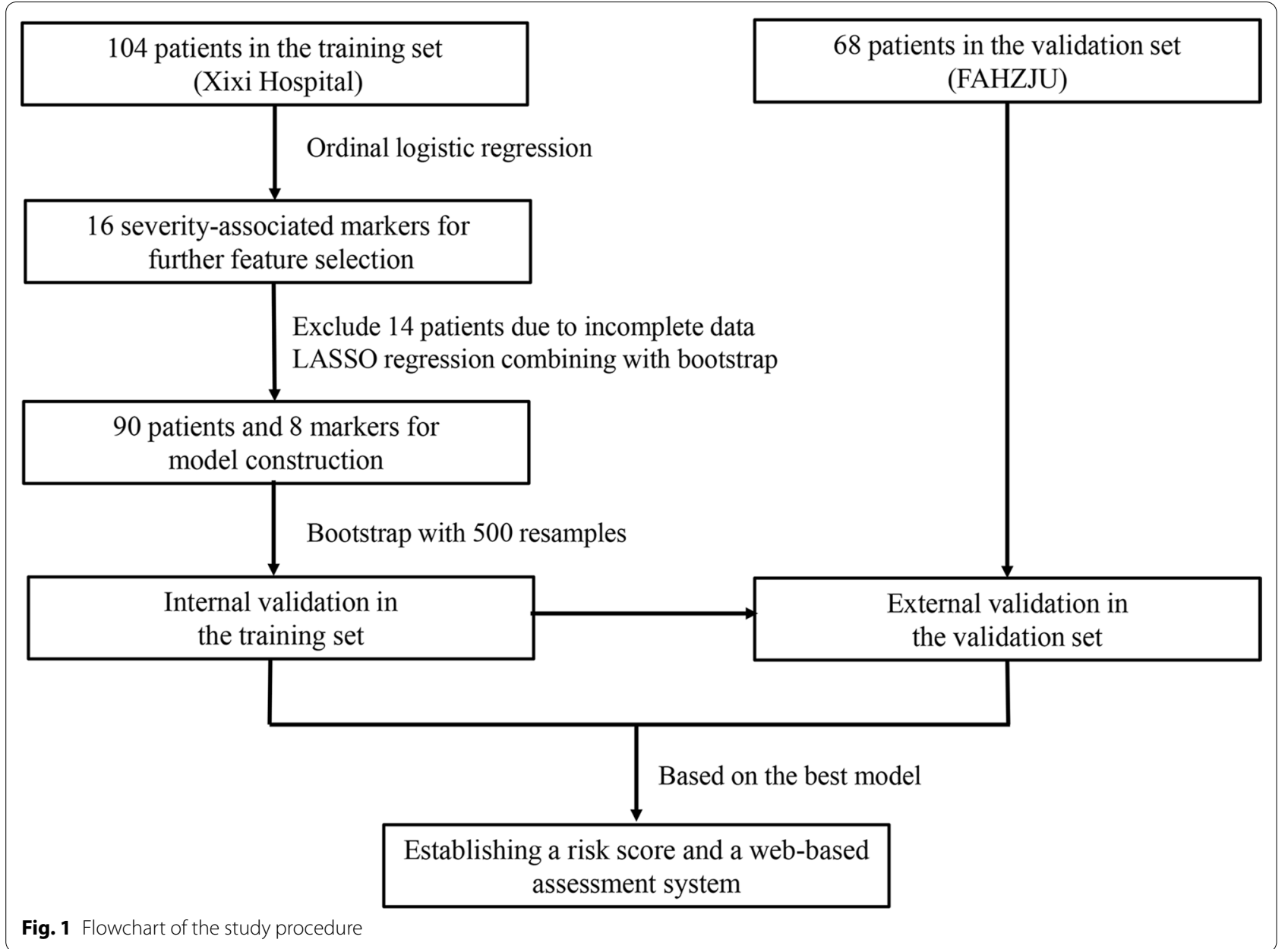

shortness of breath, diarrhoea and myalgia. Laboratory markers of laboratory tests included the following eight categories: inflammation, electrolytes, nutritional metabolism, and liver, renal, cardiac, respiratory, coagulation functions.

\section{Statistical analysis}

Continuous variables were presented as median (interquartile range $[\mathrm{IQR}]$ ), and categorical variables were presented as numbers (percentage). Continuous laboratory markers were dichotomously categorised (normal versus abnormal) under the criteria of their clinical reference values. Severity-associated markers of COVID-19 were screened using the ordinal logistic regression.

To construct an assessment model, two criteria were set for selecting markers: $\mathrm{P}$ value $<0.05$ in the ordinal logistic regression, and at least half of severe or critical patients had an abnormality in the marker. Least Absolute Shrinkage and Selection Operator (LASSO) regression was used for further feature selection. Optimal regularization parameter $(\lambda)$ was estimated by fivefold cross-validation. To increase the stability of feature selection, we used bootstrap with 1000 resamples and built a LASSO regression model for each bootstrap set. The markers, which were present in more than half of all bootstrap sets, were included in the final model.

Assessment models were constructed using logistic regression, ridge regression, support vector machine, and random forest in the training set. The performance of different models was evaluated by the area under the receiver operator characteristic curve (AUROC). For the internal validation, we used bootstrap with 500 resamples to decrease the over-fitting. For the external validation, four models were assessed in the validation set, respectively. A risk score was established according to the result of the best model. The performance of the risk score in all patients was evaluated using AUROC and calibration curve. The optimal cutoff value was calculated with the maximal Youden index. A web-based assessment system was developed based on the risk score.

All statistical analyses were conducted using $\mathrm{R}$ software, version 3.6.2 ( $\mathrm{R}$ Foundation for Statistical Computing). A two-sided $\mathrm{P}$ value $<0.05$ was considered statistically significant. 

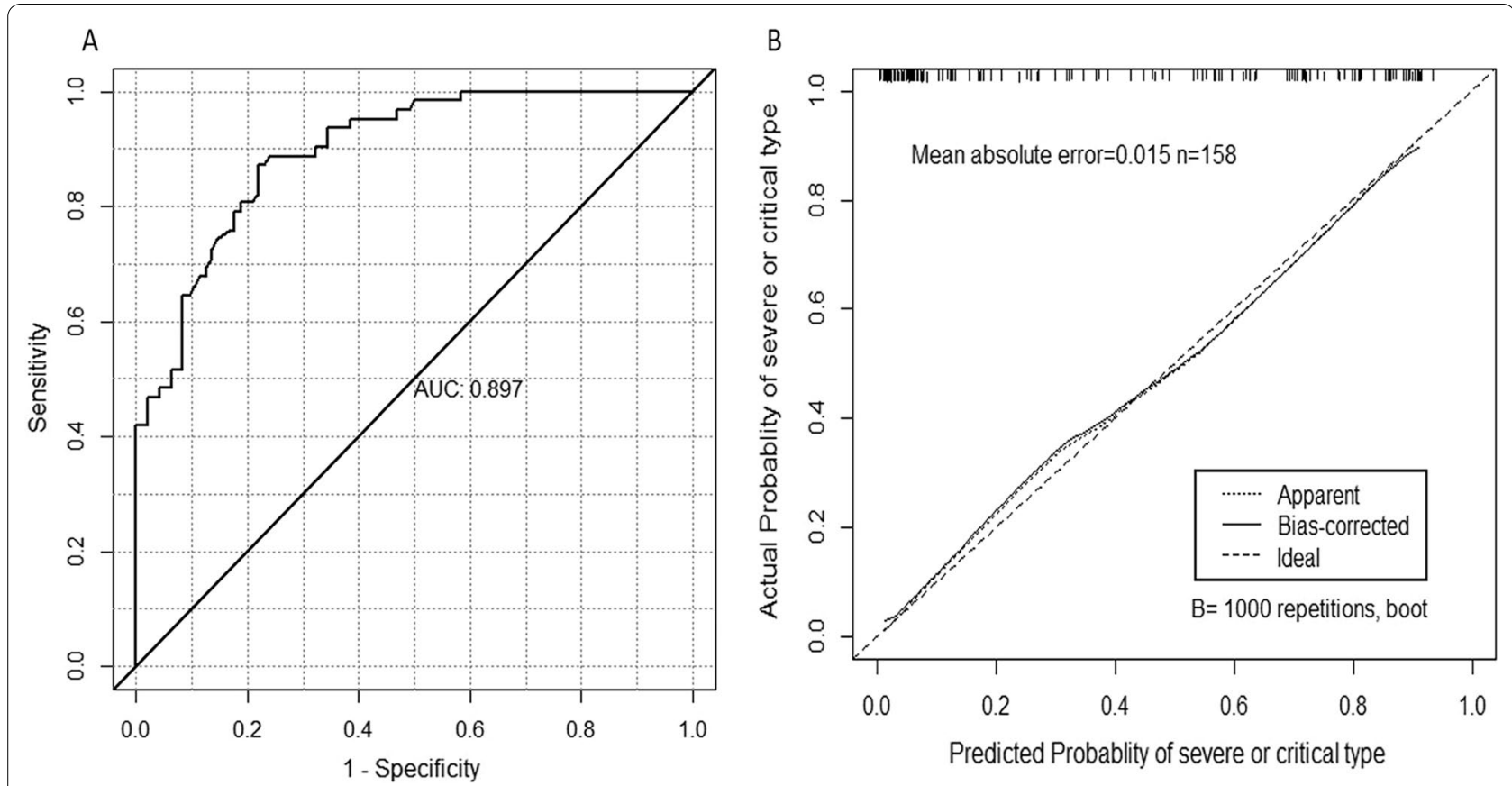

Fig. 2 The performance assessment of the risk score in all patients. A The receiver operator characteristic curve of the risk score. B The calibration curve of the risk score with bootstrap

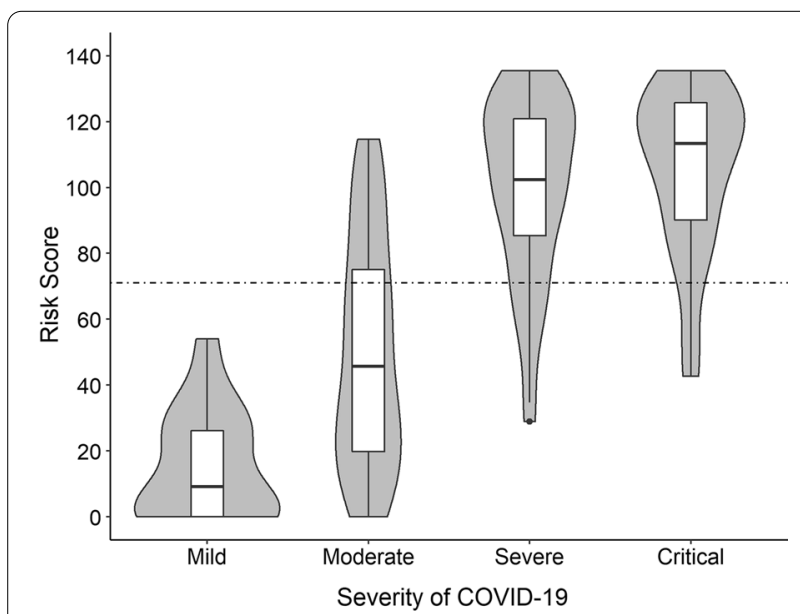

Fig. 3 Violin plots of the risk scores in different degrees of COVID-19 severity. Width of the violin plots represented the frequency of the risk score in that level. Each box represented the lower quartile (25\%), median (50\%) and upper quartile (75\%). The horizontal dashed line represented the cutoff value of the risk score (71)

\section{Results}

\section{Basic characteristics of the study population}

The flowchart of the study procedure is illustrated in Fig. 1. Basic characteristics of the COVID-19 patients are summarised in Table 1. The patients in the training set had a median age of 42.0 years (IQR: $33.0-56.5$ ) and a median BMI of $22.5 \mathrm{~kg} / \mathrm{m}^{2}$ (IQR: 20.3-25.0). Among them, 47(45.2\%) patients were men, and $23(22.1 \%)$ patients had at least one comorbidity. During hospitalisation, $21(20.2 \%)$ patients were classified as mild type, $72(69.2 \%)$ as moderate type, and $11(10.6 \%)$ as severe type. In the validation set, the median age and BMI were 59.0 years (IQR: $48.0-66.0$ ) and $24.7 \mathrm{~kg} / \mathrm{m}^{2}$ (IQR: $22.1-$ $27.0)$, respectively. $44(64.7 \%)$ patients were men, and $56(82.4 \%)$ patients had at least one comorbidity. During hospitalisation, $16(23.5 \%)$ patients were classified as moderate type, 29 (42.7\%) as severe type, and 23 (33.8\%) as critical type. The most common clinical symptoms were fever and cough, followed by expectoration and shortness of breath in both the training and validation sets (Figs. 2, 3 and 4.

\section{Severity-associated markers of COVID-19}

Table 2 presents the associations of clinical characteristics with the severity of COVID-19 in the training set. For demographic characteristics and clinical symptoms, age, comorbidity, and fever were associated with the severity of COVID-19 (all P values $<0.05$ ). For dichotomous laboratory markers, higher levels of $\mathrm{C}$-reactive protein (CRP), lactate dehydrogenase (LDH), serum amyloid A, fibrinogen (FIB), D-dimer, adenosine deaminase, reduced haemoglobin, and lower levels of lymphocyte, eosinophil, platelet counts, calcium, phosphorus, albumin (ALB), albumin/globulin, prealbumin, total cholesterol, high 


\section{浙江大学公共卫生学院 \& 杭州西溪医院}

School of Public Health Zhejiang University \& Hangzhou Xixi Hospital

\section{Calculation Tool For Predicting The Severity Of COVID-19 At Admission}

Please answer the questions below for calculation.

\begin{tabular}{|c|c|c|c|}
\hline \multicolumn{4}{|c|}{$\begin{array}{l}\text { Whether the following indicators are above the uppe } \\
\text { reference values (以下指标是否高于海床参考值上限) }\end{array}$} \\
\hline * (1) & Lactate dehydrogenase (乳酸眖氢醇) & Yes & No \\
\hline * (2) & C-reactive protein（C反应蛋白） & Yes & No \\
\hline$*(3)$ & D-dimer (D-二聚体) & Yes & No \\
\hline$*(4)$ & Fibrinogen（纤维蛋白原） & Yes & No \\
\hline \multicolumn{4}{|c|}{ Whether has any comorbidities } \\
\hline \multicolumn{2}{|c|}{ × 是否具有基础疾病 } & Yes & No \\
\hline
\end{tabular}

Whether the following indicators are below the lower limits of clinical reference values（以下指标是否低于临床参考值下限)

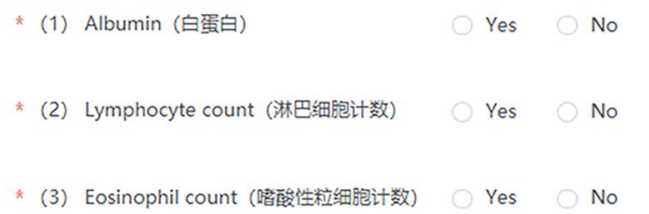

Number of abnormalities in electrolytes

*电解质异常的个效

Assessment Result（评估结果）

Total points (总分) :

Risk group at the optimal cutoff value (最佳政断值下色险分导):

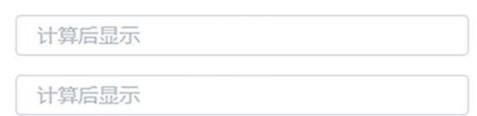

Probability for severe or critical type (重型或后重型的期丞) :

Notes (备注)：

1. Comorbidities include diabetes, hypertension, cardiovascular disease, severe congenital disease, cancer and chronic liver, renal, respiratory disease. (基础疾病包括: 糟屚病，

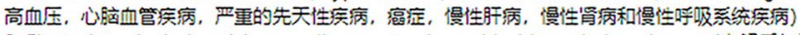

2. Electrolytes include calcium, sodium, potassium, chloride and phosphorus (电解质包括: 钧, 钠, 㳌, 氯和磷)

Fig. 4 The online calculator for assessing the severity of COVID-19 at admission. Website: http://www.gtrsp.com:8011/

density lipoprotein cholesterol, retinol binding protein, apolipoprotein $\mathrm{A} 1, \mathrm{SaO}_{2}, \mathrm{PaO}_{2} / \mathrm{FiO}_{2}$ increased the risk of elevated COVID-19 severity (all P values $<0.05$ ). Detailed results of the associations of continuous laboratory markers data with COVID-19 severity are summarised in Additional file 1: Table S1.

\section{Model construction and evaluation}

Based on the criteria described in the Methods, 18 candidate markers and 90 patients were selected for the model construction. Because of similar clinical function, D-dimer and FIB were combined into a new variable of coagulation function as DFIB. Abnormal DFIB was defined as patients with abnormal D-dimer or FIB. Electrolyte disturbance was calculated based on the sum of abnormalities in calcium, phosphorus, potassium, sodium and chlorine. Thus, 16 markers were included in LASSO regression for further feature selection. After 1000 resamples by bootstrap, ALB, CRP, LDH, DFIB, comorbidity, lymphocyte count, eosinophil count, and electrolyte disturbance were finally selected as the predictors in the model. The detailed frequency of each marker in the 1000 LASSO models is summarised in Additional file 1: Tables S2 and S3.

Table 3 presents the performance of each model in the internal and external validations. For the internal validation, high levels of AUROCs were found among four models of logistic regression, ridge regression, support vector machine, and random forest from 0.919 (95\% CI $0.793-0.955$ ) to 0.973 (95\% CI 0.935-0.993). For the external validation, the ridge regression model showed the best performance with the highest AUROC of 0.827 (95\% CI 0.716-0.921). Therefore, the ridge regression model was considered as the best model because of its high predictive power.

A risk score was then calculated according to the result of the ridge regression model using the following formula: 
Table 1 Basic characteristics of the study population

\begin{tabular}{lll}
\hline & Training set $(\mathbf{n}=\mathbf{1 0 4})$ & Validation set $(\mathbf{n}=\mathbf{6 8})$ \\
\hline $\begin{array}{l}\text { Age (years), median } \\
(\mathrm{IQR})\end{array}$ & $42.0(33.0-56.5)$ & $59.0(48.0-66.0)$ \\
$\begin{array}{l}\mathrm{BMI}\left(\mathrm{kg} / \mathrm{m}^{2}\right) \text {, median } \\
(\mathrm{IQR})\end{array}$ & $22.5(20.3-25.0)$ & $24.7(22.1-27.0)$ \\
Sex, $\mathrm{n}(\%)$ & \\
Male & & \\
Female & $47(45.2)$ & $44(64.7)$ \\
Comorbidity ${ }^{\mathrm{a}}, \mathrm{n}(\%)$ & $57(54.8)$ & $24(35.3)$ \\
Yes & & \\
No & $23(22.1)$ & $56(82.4)$ \\
Degree of severity, $\mathrm{n}(\%)$ & $81(77.9)$ & $12(17.6)$ \\
Mild & $21(20.2)$ & $0(0)$ \\
Moderate & $72(69.2)$ & $16(23.5)$ \\
Severe & $11(10.6)$ & $29(42.7)$ \\
Critical & $0(0)$ & $23(33.8)$ \\
Clinical symptoms, $\mathrm{n}(\%)$ & & \\
Fever & $69(66.3)$ & $58(85.3)$ \\
Fatigue & $6(5.8)$ & $13(19.1)$ \\
Cough & $46(44.2)$ & $42(61.8)$ \\
Expectoration & $9(8.7)$ & $28(41.2)$ \\
Shortness of breath & $8(7.7)$ & $19(27.9)$ \\
Diarrhoea & $2(1.9)$ & $7(10.3)$ \\
Myalgia & $2(1.9)$ & \\
\hline
\end{tabular}

a Comorbidity was defined as having at least one of the following diseases: diabetes, hypertension, cardiovascular disease, severe congenital disease, cancer and chronic liver, renal, respiratory disease

$I Q R$ Interquartile range; $B M I$ Body mass index severe patients (median: 102.38, IQR: 81.37-120.92). The critical patients had the highest median risk score of 113.42 (IQR: 87.89-125.75). In order to help clinicians to detect the patients who were likely to develop severe or critical COVID-19 at admission, we developed a web-based assessment system based on our risk score. (Fig. 4, Website: http://www.gtrsp.com:8011/).

\section{Discussion}

Early identification of patients who were likely to develop severe or critical COVID-19 would help reduce the casefatality rate and efficiently utilize the limited medical resources. In this study, we identified a panel of clinical markers associated with the severity of COVID-19 and constructed different severity-prediction models. We found that the ridge regression model was the best based on high AUROCs in both the internal and external validations of 0.930 (95\% CI, 0.914-0.943) and 0.827 (95\% CI, 0.716-0.921), respectively. Furthermore, we established a risk score and a web-based assessment system to help clinicians to detect the patients who were likely to develop severe or critical COVID-19 at admission.

Previous studies showed that severe or critical COVID19 patients were older, had more comorbidities, higher levels of LDH, D-dimer, CRP, and lower levels of ALB, lymphocyte count $[3-6,8]$. These findings were consistent in our study. Moreover, using the data of 208 patients from Fuyang, Anhui Province, Ji et al. [12] established a scoring model named as CALL to predict the severity of COVID-19. Dong et al. [13] also developed a scoring

Risk score $=26.78 \times$ lactate dehydrogenase $+19.31 \times \mathrm{C}-$ reactive protein $+17.16 \times$ DFIB

$+19.81 \times$ albumin $+17.59 \times$ comorbidity $+9.19 \times$ eosinophil count $+4.83 \times$ electrolyte

disturbance $+6.25 \times$ lymphocyte count

All markers, except electrolyte disturbance, were in dichotomous forms $(1=$ abnormal, $0=$ normal $)$. The range of electrolyte disturbance was from 0 to 5 . Figure 2 presents the receiver operating characteristic curve (A) and calibration curve (B) of the risk score. The risk score indicated good discrimination of severe or critical type with an AUROC of 0.897 (95\% CI 0.845-0.940). In addition, calibration curve graphically showed good consistency between the predicted and actual probabilities of severe or critical type. Using the optimal cutoff value of 71 , the sensitivity of the risk score was $87.1 \%$, and specificity was $78.1 \%$ for the COVID-19 severity prediction. Figure 3 presents the distribution of risk scores in different degrees of COVID-19 severity. The mild patients had the lowest median risk score of 9.19 (IQR: 0-26.82), then after the moderate (median: 45.65, IQR: 19.56-76.91) and system with the data of 147 patients from Wuhan, Hubei Province. The AUROCs of their models were 0.910 and 0.843 , slightly lower than the AUROC of our assessment model in the internal validation (0.930). However, their models were not validated in an external dataset, leading to the limitation of their generalizability. In contrast, our model validated in an independent dataset and obtained a satisfactory AUROC of 0.827 .

Among the eight markers in our model, LDH, CRP, ALB, and lymphocyte count were well-recognized predictors for COVID-19 severity [16]. For eosinophil count, Zhu et al. [14] demonstrated that decreased eosinophils could induce acute lung injury in the mouse model. Liu et al. [15] also found that increased eosinophil count predicted the improvement in COVID-19 progression. Several studies reported that severe or critical COVID19 patients often experienced electrolyte disturbances [3, 
Table 2 Associations of clinical characteristics with the severity of COVID-19 in the training set

\begin{tabular}{|c|c|c|c|c|c|}
\hline & Mild $^{a}$ & Moderate $^{a}$ & Severe $^{a}$ & OR $(95 \% \mathrm{CI})$ & $P$ value \\
\hline Number & 21 & 72 & 11 & & \\
\hline \multicolumn{6}{|l|}{ Demographic characteristics } \\
\hline Age (years) & $30.0(14.5-40.5)$ & $44.5(34.3-57.0)$ & $60.0(45.0-72.0)$ & $1.07(1.04-1.11)$ & $<0.001$ \\
\hline Female & $8(38.1)$ & $44(61.1)$ & $5(45.5)$ & $1.52(0.66-3.48)$ & 0.326 \\
\hline BMI $\left(\mathrm{kg} / \mathrm{m}^{2}\right)$ & $21.2(18.1-25.4)$ & $22.5(20.4-25.1)$ & $23.4(20.4-24.0)$ & $1.08(0.96-1.22)$ & 0.218 \\
\hline Comorbidity $^{b}$ & $1(4.8)$ & $15(20.8)$ & $7(63.6)$ & $8.09(2.46-26.55)$ & $<0.001$ \\
\hline \multicolumn{6}{|l|}{ Clinical symptoms } \\
\hline Fever & $10(47.6)$ & $49(68.1)$ & $10(90.9)$ & $3.11(1.26-7.66)$ & 0.014 \\
\hline Fatigue & $0(0)$ & $5(6.9)$ & $1(9.1)$ & $2.89(0.49-16.95)$ & 0.240 \\
\hline Cough & $7(33.3)$ & $34(47.2)$ & $5(45.5)$ & $1.47(0.64-3.39)$ & 0.367 \\
\hline Expectoration & $2(9.5)$ & $5(6.9)$ & $2(18.2)$ & $1.51(0.34-6.65)$ & 0.584 \\
\hline Shortness of breath & $1(4.8)$ & $6(8.3)$ & $1(9.1)$ & $1.51(0.32-7.22)$ & 0.604 \\
\hline Diarrhoea & $1(4.8)$ & $1(1.4)$ & $0(0)$ & $0.23(0.01-3.72)$ & 0.301 \\
\hline Myalgia & $1(4.8)$ & $1(1.4)$ & $0(0)$ & $0.23(0.01-3.72)$ & 0.301 \\
\hline \multicolumn{6}{|l|}{ Laboratory tests } \\
\hline WBC count $\left(<3.5 \times 10^{9} / L\right)$ & $1(4.8)$ & $5(6.9)$ & $2(18.2)$ & $2.66(0.56-12.66)$ & 0.220 \\
\hline Lymphocyte count $\left(<1.1 \times 10^{9} / \mathrm{L}\right)$ & $5(23.8)$ & $31(43.1)$ & $7(63.6)$ & $2.73(1.11-6.68)$ & 0.028 \\
\hline Eosinophil count $\left(<0.02 \times 10^{9} / \mathrm{L}\right)$ & $4(19.0)$ & $39(54.2)$ & $9(81.8)$ & $5.63(2.08-15.18)$ & 0.001 \\
\hline Platelet count $\left(<125 \times 10^{9} / \mathrm{L}\right)$ & $0(0)$ & $5(6.9)$ & $3(27.3)$ & $7.52(1.62-34.98)$ & 0.010 \\
\hline $\mathrm{CRP}(>10 \mathrm{mg} / \mathrm{L})$ & $2(9.5)$ & $30(41.7)$ & $10(90.9)$ & $12.08(3.38-43.21)$ & $<0.001$ \\
\hline $\mathrm{LDH}(>250 \mathrm{U} / \mathrm{L})$ & $0(0)$ & $10(14.1)$ & $6(54.5)$ & $11.55(3.14-42.52)$ & $<0.001$ \\
\hline $\mathrm{SAA}(>10 \mathrm{mg} / \mathrm{L})$ & $11(52.4)$ & $60(83.3)$ & $11(100)$ & $5.97(2.13-16.67)$ & 0.001 \\
\hline Calcium $(<2.2 \mathrm{mmol} / \mathrm{L})$ & $0(0)$ & $19(27.5)$ & $9(81.8)$ & $22.69(4.77-108.01)$ & $<0.001$ \\
\hline Potassium (<3.5 mmol/L) & $4(19.0)$ & $20(27.8)$ & $3(27.3)$ & $1.38(0.53-3.56)$ & 0.506 \\
\hline Phosphorus (<0.81 mmol/L) & $0(0)$ & $14(20.3)$ & $3(27.3)$ & $3.58(1.10-11.6)$ & 0.034 \\
\hline Chlorine (<99 mmol/L) & $0(0)$ & $9(12.5)$ & $2(18.2)$ & $3.36(0.85-13.24)$ & 0.083 \\
\hline Sodium (<137 mmol/L) & $6(28.6)$ & $25(34.7)$ & $7(63.6)$ & $2.17(0.89-5.28)$ & 0.090 \\
\hline $\operatorname{ALB}(<40 \mathrm{~g} / \mathrm{L})$ & $3(14.3)$ & $30(41.7)$ & $11(100)$ & 12.12(3.41-43.11) & $<0.001$ \\
\hline$A L B / G L B(<1.2)$ & $0(0)$ & $18(25.0)$ & $7(63.6)$ & $10.34(2.98-35.85)$ & $<0.001$ \\
\hline PALB (<180 mg/L for females and < $200 \mathrm{mg} / \mathrm{L}$ for males) & $7(35.0)$ & $49(74.2)$ & $11(100)$ & $7.70(2.74-21.68)$ & $<0.001$ \\
\hline $\mathrm{TG}(>1.7 \mathrm{mmol} / \mathrm{L})$ & $5(23.8)$ & $13(18.1)$ & $3(27.3)$ & $0.98(0.35-2.73)$ & 0.975 \\
\hline $\mathrm{TC}(<3 \mathrm{mmol} / \mathrm{L})$ & $0(0)$ & $3(4.2)$ & $2(18.2)$ & $7.41(1.16-47.21)$ & 0.034 \\
\hline $\mathrm{HDL}-\mathrm{C}(<1.1 \mathrm{mmol} / \mathrm{L}$ for females and $<1.04 \mathrm{mmol} / \mathrm{L}$ for males $)$ & $4(19.0)$ & $32(44.4)$ & $8(72.7)$ & $4.06(1.57-10.52)$ & 0.004 \\
\hline $\operatorname{RBP}(<23 \mathrm{mg} / \mathrm{L})$ & $5(25.0)$ & $36(54.5)$ & $8(80.0)$ & $4.20(1.60-11.03)$ & 0.004 \\
\hline Apo A1 (<1 g/L) & $10(50.0)$ & $43(65.2)$ & $10(100)$ & $3.20(1.26-8.15)$ & 0.015 \\
\hline $\mathrm{GLB}(>40 \mathrm{~g} / \mathrm{L})$ & $0(0)$ & $3(4.2)$ & $0(0)$ & $1.48(0.12-17.76)$ & 0.756 \\
\hline $\mathrm{FIB}(>3.5 \mathrm{~g} / \mathrm{L})$ & $4(19.0)$ & $30(41.7)$ & $9(81.8)$ & $5.00(1.84-13.54)$ & 0.002 \\
\hline D-dimer (>0.55 mg/L) & $2(9.5)$ & $12(16.7)$ & $6(54.5)$ & $4.91(1.57-15.41)$ & 0.006 \\
\hline $\mathrm{SaO}_{2}(\leq 93 \%)$ & $0(0)$ & $4(5.7)$ & $3(27.3)$ & $8.33(1.64-42.30)$ & 0.011 \\
\hline $\mathrm{PaO}_{2} / \mathrm{FiO}_{2}(\leq 300 \mathrm{mmHg})$ & $0(0)$ & $4(5.7)$ & $3(27.3)$ & $8.33(1.64-42.30)$ & 0.011 \\
\hline $\mathrm{PaCO}_{2}(<35 \mathrm{mmHg})$ & $1(6.2)$ & $6(8.6)$ & $3(27.3)$ & $3.43(0.82-14.41)$ & 0.093 \\
\hline ALT (> $40 \mathrm{U} / \mathrm{L}$ for females and $>50 \mathrm{U} / \mathrm{L}$ for males) & $4(19.0)$ & $6(8.3)$ & $2(18.2)$ & $0.68(0.19-2.41)$ & 0.553 \\
\hline AST (> $35 \mathrm{U} / \mathrm{L}$ for females and $>40 \mathrm{U} / \mathrm{L}$ for males) & $4(19.0)$ & $8(11.1)$ & $5(45.5)$ & $2.16(0.69-6.78)$ & 0.188 \\
\hline $\mathrm{ADA}(>15 \mathrm{U} / \mathrm{L})$ & $1(5.0)$ & $4(6.1)$ & $4(40.0)$ & $7.95(1.79-35.24)$ & 0.006 \\
\hline Y-GTP (> $45 \mathrm{U} / \mathrm{L}$ for females and $>60 \mathrm{U} / \mathrm{L}$ for males) & $2(33.3)$ & $5(12.2)$ & $1(14.3)$ & $0.43(0.08-2.46)$ & 0.344 \\
\hline Troponin T (>0.014 ng/mL) & $1(10.0)$ & $2(4.3)$ & $2(25.0)$ & $3.24(0.46-22.65)$ & 0.236 \\
\hline Serum iron( $<7.8 \mathrm{umol} / \mathrm{L}$ for females and $>10.6 \mathrm{umol} / \mathrm{L}$ for males) & $3(30.0)$ & $17(40.5)$ & $5(71.4)$ & $2.64(0.79-8.78)$ & 0.113 \\
\hline $\begin{array}{l}\text { Serum creatinine (> } 81 \mathrm{umol} / \mathrm{L} \text { for females and }>111 \mathrm{umol} / \mathrm{L} \text { for } \\
\text { males) }\end{array}$ & $0(0)$ & $4(5.6)$ & $1(9.1)$ & $3.26(0.48-21.97)$ & 0.225 \\
\hline
\end{tabular}


Table 2 (continued)

\begin{tabular}{|c|c|c|c|c|c|}
\hline & Mild $^{a}$ & Moderate $^{\mathrm{a}}$ & Severe $^{a}$ & OR $(95 \% \mathrm{Cl})$ & $P$ value \\
\hline $\begin{array}{l}\text { Urine creatinine ( }<2470 \text { umol/L for females and }<3450 \mathrm{umol} / \mathrm{L} \\
\text { for males) }\end{array}$ & $1(9.1)$ & $3(5.7)$ & $0(0)$ & $0.49(0.05-4.44)$ & 0.524 \\
\hline Reduced haemoglobin (> 7\%) & $0(0)$ & $4(5.7)$ & $3(27.3)$ & $8.33(1.64-42.30)$ & 0.011 \\
\hline Lactate (> $1.6 \mathrm{mmol} / \mathrm{L})$ & $4(25.0)$ & 14(20.0) & $3(27.3)$ & $1.01(0.35-2.94)$ & 0.982 \\
\hline $\operatorname{lgG}(>16 \mathrm{~g} / \mathrm{L})$ & $0(0)$ & $3(5.4)$ & $1(12.5)$ & $4.45(0.53-37.11)$ & 0.168 \\
\hline GPDA (<44 U/L) & $0(0)$ & $1(1.5)$ & $0(0)$ & $1.51(0.02-105.39)$ & 0.849 \\
\hline FFA (> 769 umol/L) & $1(5.0)$ & $3(4.5)$ & $0(0)$ & $0.60(0.08-4.67)$ & 0.625 \\
\hline ß2-microglobulin (> $3 \mathrm{mg} / \mathrm{L})$ & $0(0)$ & $6(8.5)$ & $1(9.1)$ & $2.65(0.51-13.83)$ & 0.248 \\
\hline $\begin{array}{l}\text { Uric acid ( }<154.7 \text { umol/L for females and }<208.3 \text { umol/L for } \\
\text { males) }\end{array}$ & $1(4.8)$ & $7(9.7)$ & $2(18.2)$ & $2.39(0.58-9.89)$ & 0.230 \\
\hline eGFR (<90 ml/min) & $1(4.8)$ & $16(22.2)$ & $3(27.3)$ & $2.66(0.89-7.96)$ & 0.079 \\
\hline DBIL (> 6.84 umol/L) & $5(23.8)$ & $15(20.8)$ & $5(45.5)$ & $1.64(0.61-4.37)$ & 0.324 \\
\hline
\end{tabular}

OR Odds ratio, $C I$ Confidence interval, $B M I$ Body mass index, WBC White blood cell, $C R P C$-reactive protein, $L D H$ Lactate dehydrogenase, SAA Serum amyloid $\mathrm{A}$, ALB Albumin, ALB/GLB Albumin/Globulin, TC Total cholesterol, HDL-C High density lipoprotein cholesterol, PALB Prealbumin; RBP Retinol binding protein, Apo A1

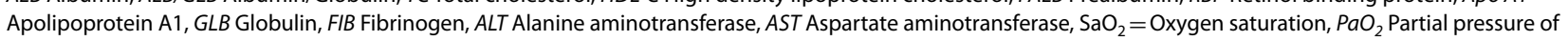
oxygen in arterial blood, $\mathrm{FiO}_{2}$ Inspired oxygen fraction, ADA Adenosine deaminase; $\mathrm{PaCO} 2$ Partial pressure of carbon dioxide, $\gamma$-GTP $\gamma$-glutamyltranspeptidase, IgG Immunoglobulin G, GPDA = Glycyl-proline-dipeptidyl aminopeptidase, FFA Free fatty acids, eGFR Estimated glomerular filtration rate, DBIL Direct bilirubin

${ }^{a}$ Data were presented as median (IQR), or $\mathrm{n}(\%)$ where appropriate

${ }^{b}$ Comorbidity was defined as having at least one of the following diseases: diabetes, hypertension, cardiovascular disease, severe congenital disease, cancer and chronic liver, renal, respiratory disease

Table 3 Performance of different models in the internal and external validations

\begin{tabular}{lllll}
\hline & Logistic regression & Ridge regression & Random forest & Support vector machine \\
\hline $\begin{array}{c}\text { Internal validation } \\
\text { AUROC }(95 \% \mathrm{Cl})\end{array}$ & $0.919(0.793-0.955)$ & $0.930(0.914-0.943)$ & $0.973(0.935-0.993)$ & $0.955(0.892-0.993)$ \\
$\begin{array}{c}\text { External validation } \\
\text { AUROC }(95 \% \mathrm{Cl})\end{array}$ & $0.756(0.617-0.869)$ & $0.827(0.716-0.921)$ & $0.795(0.669-0.905)$ & $0.802(0.682-0.908)$ \\
\hline
\end{tabular}

AUROC Area under the receiver operator characteristic curve, $C I$ Confidence interval

$4,16]$. In our study, we used the sum of abnormalities in potassium, calcium, sodium, phosphorus and chlorine to comprehensively evaluate the degree of electrolyte disturbances. D-dimer and FIB were indicators of coagulation function. Chen et al. [8] reported that patients infected with SARS-CoV-2 had abnormal coagulation function (hypercoagulation). We combined the two indicators to increase the sensitivity of judging abnormal coagulation and avoid the collinearity of the two markers. Different from other studies, age was not included in our final model. This might be owing to the high correlation between age and comorbidity in the training set, and the LASSO regression identified comorbidity as a more important marker.

There were several limitations in our study. First, there were different distribution on the severity of COVID-19 between the training and validation sets. There were no critical cases in training set while without mild cases in validation set. This difference was due to the rule of government on the COVID-19 prevention and control in Zhejiang Province in China. Xixi Hospital (municipal-level hospital for infectious diseases) mainly receive and cure the patients with mild, moderate, and severe COVID-19 (no critical patients), while FAHZJU (provincial-level hospital) is mainly responsible for moderate, severe, and critical patients (no mild patients). The different distribution of the severity might have influences on the model construction and validation. However, even there were these differences, the ideal performance was still obtained in the validation stage, and this result indicated that there was relatively high generalizability in our model. Second, the subjects were mainly recruited from Hangzhou and the sample size was relatively small. This would limit the generalizability of our model. Additional validation from areas outside Zhejiang should be conducted in the future. Third, because of the retrospective study design, some laboratory tests were not done in some patients. Therefore, their associations with the severity of COVID-19 might be 
misestimated. Fourth, the clinical data of the subjects were not comprehensive. Adding other specific markers such as cytokines might improve the performance of our model. Finally, due to the low prevalence of comorbidity, the risks in different types of comorbidities were not considered in the assessment model.

\section{Conclusions}

In this study, we screened eight severity-associated clinical markers of lactate dehydrogenase, C-reactive protein, albumin, comorbidity, electrolyte disturbance, coagulation function, eosinophil and lymphocyte counts in COVID-19 patients. Based on these eight markers, an assessment model was constructed to help the clinician to evaluate the likelihood of developing severity of COVID-19 at admission and early take measures on clinical treatment.

\begin{abstract}
Abbreviations
ALB: Albumin; AUROC: Area under the receiver operator characteristic curve; BMI: Body mass index; CT: Computerized tomography; Cl: Confidence interval; COVID-19: Coronavirus disease 2019; CRP: C-reactive protein; FIB: Fibrinogen; $\mathrm{FiO}_{2}$ : Inspired oxygen fraction; IQR: Interquartile range; LASSO: Least Absolute Shrinkage and Selection Operator; LDH: Lactate dehydrogenase; OR: Odds ratio; $\mathrm{PaO}_{2}$ : Partial pressure of oxygen in arterial blood; $\mathrm{SaO}_{2}$ : Oxygen saturation; SARS-CoV-2: Severe acute respiratory syndrome coronavirus 2; WHO: World Health Organization.
\end{abstract}

\section{Supplementary Information}

The online version contains supplementary material available at https://doi. org/10.1186/s12879-021-06509-6.

Additional file 1: Table S1. Associations of continuous laboratory markers with the severity of COVID-19 in the training set. Table S2. Remaining frequency of the 16 markers in 1000 LASSO regression models. Table S3. Associations of the selected eight markers with the severity of COVID-19 in the external validation set.

\section{Acknowledgements}

Not applicable.

\section{Authors' contributions}

$Y Z, J Q, J H$, and SL conceived the study and design. JQ, SL, JH, DY, WM, HC, FW, $\mathrm{CL}, J W, K Q, R W$ had role in data collecting. $Y Z, J Q, D H, J X$, ZL were responsible of DNA analysis. HM, JH, JT, JX, and JS had roles in the study design, data analysis and interpretation. $Y Z, J Q, D H, M W$ had roles in writing. All the authors have read the manuscript and agreed to publish. All authors read and approved the final manuscript.

\section{Funding}

This work was partially supported by the special funding of New Coronary Pneumonia, Institute of China's System Research, Zhejiang University (YQYB2006). They had no role in the design of this study, data collection, data analysis, data interpretation and writing the manuscript.

\section{Availability of data and materials}

The datasets used and/or analyzed during the current study are available from the corresponding authors on reasonable request.

\section{Declarations}

\section{Ethics approval and consent to participate}

This study was approved by the Ethics Committee of Hangzhou Xixi Hospital (2020-24) and the First Affiliated Hospital, School of Medicine, Zhejiang University (FAHZJU) (2020-149). Individual informed consent was waived by the ethics committee listed above because this study used currently existing sample collected during the course of routine medical care and did not pose any additional risks to the patients. All patient data were anonymized prior to the analysis.

\section{Consent for publication}

Not applicable.

\section{Competing interests}

The authors declare that they have no competing interests.

\section{Author details}

${ }^{1}$ Hangzhou Xixi Hospital, Hangzhou 310023, Zhejiang, China. ${ }^{2}$ Department of Epidemiology \& Biostatistics, School of Public Health, Zhejiang University, Hangzhou 310058, Zhejiang, China. ${ }^{3}$ The First Affiliated Hospital, School of Medicine, Zhejiang University, Hangzhou 310003, Zhejiang, China. ${ }^{4}$ Department of Pathology, Zhejiang University School of Medicine, Hangzhou 310058, Zhejiang, China.

Received: 22 July 2020 Accepted: 30 July 2021

Published online: 09 August 2021

\section{References}

1. Zhu N, Zhang D, Wang W, Li X, Yang B, Song J, Zhao X, Huang B, Shi W, Lu R, et al. A novel coronavirus from patients with pneumonia in China, 2019. N Engl J Med. 2020;382(8):727-33.

2. World Health Organization: Novel Coronavirus (2019-nCoV). Situation report-133.2020. https://www.who.int/docs/default-source/coronaviruse/ situation-reports/20200701-covid-19-sitrep-163.pdf?sfvrsn=9a56f2ac_4. Accessed 2 Jul 2020

3. Wu ZY, McGoogan JM. Characteristics of and important lessons from the coronavirus disease 2019 (COVID-19) outbreak in China summary of a report of 72314 cases from the Chinese Center for Disease Control and Prevention. JAMA. 2020;323(13):1239-42.

4. Huang C, Wang Y, Li X, Ren L, Zhao J, Hu Y, Zhang L, Fan G, Xu J, Gu X, et al. Clinical features of patients infected with 2019 novel coronavirus in Wuhan. China Lancet. 2020;395(10223):497-506.

5. Guan WJ, Ni ZY, Hu Y, et al. Clinical characteristics of coronavirus disease 2019 in China. N Engl J Med. 2020;382:1708-20.

6. Xu XW, Wu XX, Jiang XG, Xu KJ, Ying LJ, Ma CL, Li SB, Wang HY, Zhang $\mathrm{S}, \mathrm{Gao} H N$, et al. Clinical findings in a group of patients infected with the 2019 novel coronavirus (SARS-Cov-2) outside of Wuhan, China: retrospective case series. BMJ. 2020;368:m606.

7. Wang DW, Hu B, Hu C, Zhu FF, Liu X, Zhang J, Wang BB, Xiang H, Cheng ZS, Xiong Y, et al. Clinical characteristics of 138 hospitalized patients with 2019 novel coronavirus-infected pneumonia in Wuhan. China JAMA. 2020;323(11):1061-9.

8. National Health Commission of the People's Republic of China: Chinese management guideline for COVID-19 (version 7.0) [in Chinese]. http:// www.nhc.gov.cn/yzygj/s7653p/202003/46c9294a7dfe4cef80dc7f591 2eb1989/files/ce3e6945832a438eaae415350a8ce964.pdf. Accessed 15 Mar 2020.

9. Chen NS, Zhou M, Dong X, Qu JM, Gong FY, Han Y, Qiu Y, Wang JL, Liu $Y$, Wei $Y$, et al. Epidemiological and clinical characteristics of 99 cases of 2019 novel coronavirus pneumonia in Wuhan, China: a descriptive study. Lancet. 2020;395(10223):507-13.

10. Chen ZH, Li YJ, Wang XJ, et al. Chest CT of COVID-19 in patients with a negative first RT-PCR test: Comparison with patients with a positive first RT-PCR test. Medicine (Baltimore). 2020;99(26):e20837.

11. Chen Z, Fan H, Cai J, et al. High-resolution computed tomography manifestations of COVID-19 infections in patients of different ages. Eur J Radiol. 2020;126:108972. 
12. Zheng S, Fan J, Yu F, et al. Viral load dynamics and disease severity in patients infected with SARS-CoV-2 in Zhejiang province, China, JanuaryMarch 2020: retrospective cohort study. BMJ. 2020;369:m1443.

13. World Health Organization: Clinical management of severe acute respiratory infection when novel coronavirus (2019-nCoV) infection is suspected: interim guidance. 2020. https://www.who.int/docs/defaultsource/coronaviruse/clinical-management-of-novel-cov.pdf. Accessed 15 Mar 2020

14. Ji D, Zhang $D, X u$ J, et al. Prediction for progression risk in patients with COVID-19 pneumonia: the CALL score. Clin Infect Dis. 2020. https://doi. org/10.1093/cid/ciaa414.

15. Dong $Y$, Zhou H, Li M, et al. A novel simple scoring model for predicting severity of patients with SARS-CoV-2 infection. Transbound Emerg Dis. 2020. https://doi.org/10.1111/tbed.13651.

16. Zhu C, Weng QY, Zhou LR, et al. Homeostatic and early recruited CD101_eosinophils suppress endotoxin-induced acute lung injury. Eur Respir J. 2020. https://doi.org/10.1183/13993003.02354-2019.
17. Sun S, Cai X, Wang H, et al. Abnormalities of peripheral blood system in patients with COVID-19 in Wenzhou. China Clin Chim Acta. 2020:507:174-80.

18. Liu F, Xu A, Zhang Y, et al. Patients of COVID-19 may benefit from sustained Lopinavir-combined regimen and the increase of Eosinophil may predict the outcome of COVID-19 progression. Int J Infect Dis. 2020:95:183-91.

19. Lippi $G$, South AM, Henry BM. Electrolyte imbalances in patients with severe coronavirus disease 2019 (COVID-19). Ann Clin Biochem. 2020;57(3):262-5.

\section{Publisher's Note}

Springer Nature remains neutral with regard to jurisdictional claims in published maps and institutional affiliations.
Ready to submit your research? Choose BMC and benefit from:

- fast, convenient online submission

- thorough peer review by experienced researchers in your field

- rapid publication on acceptance

- support for research data, including large and complex data types

- gold Open Access which fosters wider collaboration and increased citations

- maximum visibility for your research: over $100 \mathrm{M}$ website views per year

At BMC, research is always in progress.

Learn more biomedcentral.com/submissions 